\title{
INTEGRAL REPRESENTATIONS AND THE COMPLEX MONGE-AMPÈRE EQUATION IN STRICTLY CONVEX DOMAINS
}

\author{
NANCY K. STANTON ${ }^{1}$
}

\begin{abstract}
We prove a relationship between Aizenberg's integral representation formula for holomorphic functions in a strictly convex domain and the complex Monge-Ampère equation.
\end{abstract}

Let $\Omega$ be a bounded strictly convex domain in $\mathbf{C}^{n}, n>1$, with a smooth boundary. Let $r$ be a $e^{\infty}$ defining function for $\Omega$, so $\Omega=\{z: r(z)<0\}$ and $d r \neq 0$ on $b \Omega$. If $z \in \Omega$ and $\zeta \in b \Omega$ then $\Sigma r_{i}(\zeta)\left(\zeta_{i}-z_{i}\right) \neq 0$, since $\Omega$ lies on one side of the tangent space to $b \Omega$ at $\zeta$. Here $r_{i}=\partial r / \partial z_{i}, r_{i}=\partial r / \partial \bar{z}_{i}$, etc.

Aizenberg [2] proved that if $f$ is holomorphic on $\Omega$ and continuous on $\bar{\Omega}$, one has the following integral representation formula for $f$ :

$$
f(z)=\left(\frac{1}{2 \pi i}\right)^{n}(-1)^{(n-1)(n-2) / 2} \int_{b \Omega} \frac{f(\zeta) \partial r(\zeta) \wedge(\partial \bar{\partial} r(\zeta))^{n-1}}{\left(\Sigma r_{i}(\zeta)\left(\zeta_{i}-z_{i}\right)\right)^{n}} .
$$

(This way of writing the formula is due to Norguet [4, Theorem 11].) The expression

$$
K_{r}(\zeta, z)=\frac{\partial r(\zeta) \wedge(\partial \bar{\partial} r(\zeta))^{n-1}}{\left(\Sigma r_{i}(\zeta)\left(\zeta_{i}-z_{i}\right)\right)^{n}}
$$

is an example of a Cauchy-Fantappiè form [4].

Let $J(r)$ denote the Levi determinant,

$$
J(r)=(-1)^{n} \operatorname{det}\left[\begin{array}{c}
r r_{\bar{j}} \\
r_{i} r_{i j}
\end{array}\right] .
$$

THEOREM 1. Let do denote the induced volume element on $b \Omega$. Then formula (1) can be rewritten as

$$
f(z)=(-1)^{(n-1)(n-2) / 2} \frac{(n-1) !}{\pi^{n}} \int_{b \Omega} \frac{f(\zeta)(J(r))(\zeta) d \sigma(\zeta)}{|d r(\zeta)|\left(\sum r_{i}(\zeta)\left(\zeta_{i}-z_{i}\right)\right)^{n}} .
$$

REMARK. If the numerator of $K_{r}$ is expanded as a linear combination of the $d \zeta_{i}$ and $d \bar{\zeta}_{i}$, then for $\zeta \in b \Omega$, the coefficients are certain minors of $J(r)$ [2].

Proof OF THEOREM 1. The restriction of the form $\partial r \wedge(\partial \bar{\partial} r)^{n-1}$ to $b \Omega$ is a multiple of $d \sigma, i^{*}\left(\partial r \wedge(\partial \bar{\partial} r)^{n-1}\right)=g d \sigma$, where $i: b \Omega \rightarrow \mathbf{C}^{n}$ is inclusion. Since $(d r /|d r|) \wedge d \sigma$ is the Euclidean volume element $d v$ at points of $b \Omega$,

Received by the editors August 14, 1978.

AMS (MOS) subject classifications (1970). Primary 53C65, 32A25.

'Research supported in part by NSF grant MCS 76-08478. 


$$
(d r /|d r|) \wedge\left(\partial r \wedge(\partial \bar{\partial} r)^{n-1}\right)=(\bar{\partial} r /|d r|) \wedge \partial r \wedge(\partial \bar{\partial} r)^{n-1}=g d v .
$$

Let $\mu$ be the $2 n$ form $\mu=J(r) d v$. We will show that $\bar{\partial} r \wedge \partial r \wedge(\partial \bar{\partial} r)^{n-1}=$ $(2 i)^{n}(n-1) ! \mu$. Then $g=\left(\left((2 i)^{n}(n-1) !\right) /|d r|\right) J(r)$ and formula (3) follows from (1). Fix $\zeta_{0} \in b \Omega$ and let $\psi$ be a biholomorphic map defined in a neighborhood of $\zeta_{0}$. Since $\psi$ is biholomorphic,

$$
\psi^{*}\left(\bar{\partial} r \wedge \partial r \wedge(\partial \bar{\partial} r)^{n-1}\right)=\bar{\partial}(r \circ \psi) \wedge \partial(r \circ \psi) \wedge(\partial \bar{\partial}(r \circ \psi))^{n-1} .
$$

A simple calculation shows that $J(r \circ \psi)(\zeta)=|(\partial \psi / \partial z)(\zeta)|^{2} J(r)(\psi(\zeta))$. Hence, $\psi^{*}(\mu(\psi(\zeta)))=(J(r \circ \psi) d v)(\zeta)$. Thus, it suffices to show that, for an appropriate choice of $\psi$ with $\psi\left(\zeta_{0}\right)=\zeta_{0}$,

$$
\begin{aligned}
\left(\bar{\partial}(r \circ \psi) \wedge \partial(r \circ \psi) \wedge(\partial \bar{\partial}(r \circ \psi))^{n-1}\right)\left(\zeta_{0}\right) \\
\quad=(2 i)^{n}(n-1) ! J(r \circ \psi)\left(\zeta_{0}\right) d v\left(\zeta_{0}\right) .
\end{aligned}
$$

We can choose $\psi$ so that $(r \circ \psi)_{i}\left(\zeta_{0}\right)=0$ for $1<i<n-1,(r \circ \psi)_{n}\left(\zeta_{0}\right)=a$ $\neq 0$ and $(r \circ \psi)_{i j}\left(\zeta_{0}\right)=\lambda_{i} \delta_{i j}$ for $1<i, j<n-1$. Then the left-hand side of (4) is

$|a|^{2} d \bar{\zeta}_{n} \wedge d \zeta_{n} \wedge\left(\sum_{i=1}^{n-1} \lambda_{i} d \zeta_{i} \wedge d \bar{\zeta}_{i}\right)^{n-1}=-(-2 i)^{n}(n-1) !|a|^{2} \lambda_{1} \cdots \lambda_{n-1} d v$.

Since $J(r \circ \psi)\left(\zeta_{0}\right)=(-1)^{n-1}|a|^{2} \lambda_{1} \cdots \lambda_{n-1}$, (4) holds.

COROLLARY 2. Let $r$ be a defining function for $\Omega$ which is an approximate solution to the Monge-Ampere equation, i.e., $J(r)=1+O\left(r^{2}\right)$. Then if $f$ is holomorphic on $\Omega$ and continuous on $\bar{\Omega}$,

$$
f(z)=(-1)^{(n-1)(n-2) / 2} \frac{(n-1) !}{\pi^{n}} \int_{b \Omega} \frac{f(\zeta) d \sigma(\zeta)}{|d r(\zeta)|\left(\sum r_{i}(\zeta)\left(\zeta_{i}-z_{i}\right)\right)^{n}} .
$$

By Fefferman's work on the Monge-Ampère equation [3], we can always find such an $r$ and it is uniquely determined through first order along $b \Omega$. Approximate solutions to the Monge-Ampère equation are closely related to the geometry of $b \Omega[3]$.

Although making such a choice of $r$ simplifies formula (3), the kernel $K_{r}$ turns out to be independent of the choice of $r$.

THEOREM 3. Let $r$ and $r^{\prime}$ be defining functions for $\Omega$ and let $i: b \Omega \rightarrow \mathrm{C}^{n}$ be the inclusion map. Then $i_{\xi}^{*} K_{r}(\zeta, z)=i_{\xi}^{*} K_{r}(\zeta, z)$.

Proof. There is a function $h$ which does not vanish on $b \Omega$ such that $r^{\prime}=h r$. On $b \Omega, \partial r^{\prime}=h \partial r$ and $\partial \bar{\partial} r^{\prime}=h \partial \bar{\partial} r+\partial h \wedge \bar{\partial} r+\partial r \wedge \bar{\partial} h$. Hence, on $b \Omega$,

$$
\partial r^{\prime} \wedge\left(\partial \bar{\partial} r^{\prime}\right)^{n-1}=h^{n} \partial r \wedge(\partial \bar{\partial} r)^{n-1}+\nu \wedge \partial r \wedge \bar{\partial} r
$$

where $\nu$ is a $2 n-3$ form. Since $\partial r \wedge \bar{\partial} r=d r \wedge \bar{\partial} r, i^{*}(\nu \wedge \partial r \wedge \bar{\partial} r)=0$. Thus $i^{*}\left(\partial r^{\prime} \wedge\left(\partial \bar{\partial} r^{\prime}\right)^{n-1}\right)=h^{n_{i}^{*}}\left(\partial r \wedge(\partial \bar{\partial} r)^{n-1}\right)$. Since $\sum r_{i}^{\prime}(\zeta)\left(\zeta_{i}-z_{i}\right)=$ $h(\zeta) \sum r_{i}(\zeta)\left(\zeta_{i}-z_{i}\right)$ for $\zeta \in b \Omega$, it follows from (2) that $i_{\zeta}^{*} K_{r}(\zeta, z)=$ $i_{\xi}^{*} K_{r}(\zeta, z)$. 
REMARK. It follows immediately from Corollary 2 and Theorem 3 that if one writes Aizenberg's kernel as $\left(H(\zeta, z) /(\Phi(\zeta, z))^{n}\right) d \sigma(\zeta)$, where $H(\zeta, z)=$ $c J(r)(\zeta) /|\operatorname{dr}(\zeta)|$ and $\Phi(\zeta, z)=\Sigma r_{i}(\zeta)\left(\zeta_{i}-z_{i}\right)$, then $H(\zeta, z) \neq 0$ for $\zeta \in b \Omega$. In strictly pseudoconvex domains, Henkin's kernels can also be written in the form $\left(H(\zeta, z) /(\Phi(\zeta, z))^{n}\right) d \sigma(\zeta)$ for certain functions $H$ and $\Phi$. In this case, it is still true that $H(\zeta, \zeta) \neq 0$ for $\zeta \in b \Omega$ [1]. However, the proof is quite difficult because $H$ is not given by an explicit formula.

\section{BIBLIOGRAPHY}

1. P. Ahern and R. Schneider, The boundary behavior of Henkin's kernel, Pacific J. Math. 66 (1976), 9-14.

2. L. A. Aizenberg, Integral representations of functions which are holomorphic in convex regions of $C^{n}$ space, Dokl. Akad. Nauk SSSR 151 (1963), 1247-1249; English. transl.,Soviet Math. Dokl. 4 (1963), 1149-1152.

3. C. L. Fefferman, Monge-Ampère equations, the Bergman kernel and geometry of pseudoconvex domains, Ann. of Math. 103 (1976), 395-416.

4. F. Norguet, Introduction aux fonctions de plusieurs variables complexes: représentations intégrales, Fonctions de Plusieurs Variables Complexes, 1-97, Lecture Notes in Math., no. 409, Springer-Verlag, Berlin and New York, 1974.

Department of Mathematics, Columbia Untversity, New York, New York 10027 